\title{
The Nuclear Pseudospin Symmetry Along an Isotopic Chain
}

\author{
R. Lisboa, M. Malheiro, \\ Instituto de Física, Universidade Federal Fluminense, Nitéroi 24210-340, RJ, Brazil
}

\author{
and P. Alberto \\ Departamento de Física and Centro de Física Computacional, Universidade de Coimbra, P-3004-516 Coimbra Portugal
}

Received on 15 August, 2003.

\begin{abstract}
We investigate the isospin dependence of pseudospin symmetry in the chain of tin isotopes (from ${ }^{120} \mathrm{Sn}$ until ${ }^{170} \mathrm{Sn}$ ). Using a Woods-Saxon parametrization of the nuclear potential for these isotopes we study in detail the effect of the vector-isovector $\rho$ and Coulomb potentials in the energy splittings of neutron and proton pseudospin partners in the isotopic chain. We conclude that the realization of nuclear pseudospin symmetry does not change considerably with the mass number, and is always favored for neutrons. We also find that the $\rho$ potential accounts for essentially all the pseudospin isospin asymmetry observed and that the Coulomb potential plays a negligible role in this asymmetry. This can be explained by the dynamical nature of pseudospin symmetry in nuclei, namely the dependence of the pseudospin splittings on the shape of the nuclear mean-field potential.
\end{abstract}

\section{Introduction}

Pseudospin was introduced in the late 60's [1, 2] to account for the quasi-degeneracy of single-nucleon states with quantum numbers $(n, \ell, j=\ell+1 / 2)$ and $(n-1, \ell+2, j=\ell+$ $3 / 2$ ) where $n, \ell$, and $j$ are the radial, the orbital, and the total angular momentum quantum numbers, respectively. These levels have the same "pseudo" orbital angular momentum quantum number, $\tilde{\ell}=\ell+1$, and "pseudo" spin quantum number, $\tilde{s}=1 / 2$.

The pseudospin symmetry has been analyzed in the framework of non-relativistic models by several authors $[3,5,4]$. The subject was revived in recent years when Ginocchio [6] presented a relativistic interpretation for this symmetry showing that the quantum number $\tilde{\ell}$ is the orbital quantum number of the lower component of the Dirac spinor for spherical potentials. Moreover, he showed that $\ell$ is a good quantum number in a relativistic theory for the nucleon with scalar $S$ and vector $V$ potentials with opposite signs and the same magnitude, i.e., $\Sigma=S+V=0$. Actually, this condition can be relaxed to demand that only the derivative of $\Sigma$ be zero [7], but, if $\Sigma$ goes to zero at infinity, both conditions are equivalent.

Unfortunately, neither of these two conditions are met in nuclei. Some recent works indicate that the nuclear pseudospin symmetry has a dynamical character. It was shown that the almost degeneracy of the pseudopsin doublets not only depends in the shape of the nuclear mean fields [8] but in fact arises from a significant cancellation among the terms that contribute to the energy and not only from the pseudospin orbit interaction $[9,10]$. The non-perturbative nature of this interaction has been discussed in $[11,12]$, where it also was pointed out the dynamical character of the nuclear pseudospin.
We have investigated recently in a quantitative way the effect of the Coulomb and the vector-isovector $\rho$ potentials in the proton and neutron asymmetry seen in the nuclear pseudospin $[13,14]$. We analyzed the effect of these potentials in each of the terms that contribute to the pseudospin energy splitting. We concluded that the isospin asymmetry seen in the nuclear pseudospin is also a manifestation of the dynamical character of this symmetry.

In this paper we will present new results concerning the isospin dependence of the nuclear pseudospin along Sn isotopic chain. We will plot the energy splitting for some pseudospin doublets as a function of the mass number $A$, analyzing separately the neutron and proton levels. To this end, we solve the Dirac equation for the nucleons using a parametrization for the nuclear potential along this chain which was developed by us in a recent work [14]. That parametrization was obtained by a fit to a sophisticated selfconsistent relativistic mean field calculation [15]. We will show that the pseudospin quasi-degeneracy has a small dependence with $A$ in accordance with [16].

The symmetry is better realized for neutrons than is for protons. Furthermore, as found in previous works [8, 14], we also show that the main reason for the difference between the energy splitting of proton and neutron pseudospin partners comes from the vector-isovector potential and not from the Coulomb potential.

The paper is organized as follows: in section II we present the nuclear potentials as combinations of WoodsSaxon potentials and the respective parameters as functions of $A$ and $N-Z$. In section III we analyze the energy splittings of two pseudospin doublets that are close to the Fermi sea, for neutrons and protons, as a function of $A$ from 120 to 170 . In particular, we look for the effect of the Coulomb and vector-isovector potentials in those energy splittings. Finally, we draw our conclusions. 


\section{Dirac equation and pseudospin symmetry}

The Dirac equation for a particle of mass $m$ in external scalar $S$ and vector $V$ potentials is given by

$$
H \Psi=[\boldsymbol{\alpha} \cdot \mathbf{p}+\beta(m+S)+V] \Psi=\epsilon \Psi,
$$

where $\boldsymbol{\alpha}$ and $\beta$ are the usual Dirac matrices. The Hamiltonian in Eq. (1) is invariant under SU(2) transformations when $S=V$ or $S=-V[17,18,19]$. The second case corresponds to the realization of pseudospin symmetry. As referred before, this symmetry is related to the orbital quantum number of the lower component of the Dirac spinor.

If $S$ and $V$ are radial potentials, equation (1) gives rise to two differential equations for the upper and lower radial wave functions. Defining $\Delta=V-S, \Sigma=V+S$, and the binding energy $E=\epsilon-m$, these are

$$
\begin{array}{r}
\frac{1}{r^{2}} \frac{d}{d r}\left(r^{2} \frac{d G_{\kappa}}{d r}\right)-\frac{\ell(\ell+1)}{r^{2}} G_{\kappa}+\frac{\Delta^{\prime}}{E+2 m-\Delta} \\
\left(G_{\kappa}^{\prime}+\frac{1+\kappa}{r} G_{\kappa}\right)+(E+2 m-\Delta)(E-\Sigma) G_{\kappa}=0 \\
\frac{1}{r^{2}} \frac{d}{d r}\left(r^{2} \frac{d F_{\kappa}}{d r}\right)-\frac{\tilde{\ell}(\tilde{\ell}+1)}{r^{2}} F_{\kappa}+\frac{\Sigma^{\prime}}{E-\Sigma} \\
\left(F_{\kappa}^{\prime}+\frac{1-\kappa}{r} F_{\kappa}\right)+(E+2 m-\Delta)(E-\Sigma) F_{\kappa}=0
\end{array}
$$

where $\kappa$ is the quantum number defined by

$$
\kappa=\left\{\begin{array}{cl}
-(\ell+1) & j=\ell+\frac{1}{2} \\
\ell & j=\ell-\frac{1}{2}
\end{array} .\right.
$$

The term with $1-\kappa$ in Eq. (3) is the pseudospin-orbit term [20]. From that equation one sees that, should it be possible to set $\Sigma^{\prime}=0, \tilde{\ell}$ would be a good quantum number. Since the sign of $\kappa$ determines whether one has parallel or antiparallel spin and $\tilde{\ell}=\ell-\kappa /|\kappa|$, one sees that pairs of states with $\kappa=-(\ell+1)$ and $\kappa=\ell+2$ have the same $\tilde{\ell}=\ell+1$, the quantum numbers of the pseudospin partners. For example, for $\left[n s_{1 / 2},(n-1) d_{3 / 2}\right]$ one has $\tilde{l}=1$, for $\left[n p_{3 / 2},(n-1) f_{5 / 2}\right]$ onde has $\tilde{l}=2$ etc. Pseudospin symmetry is exact when doublets with $j=\tilde{l} \pm \tilde{s}$ are degenerate.

\section{The nuclear potential for the $\mathrm{Sn}$ iso- topic chain}

In a recent work we found a general parametrization in terms of Woods-Saxon potentials for the binding potential $\Sigma$ in the whole Sn isotopic chain. The procedure used to extract the parameters is explained in detail in [14]. We separated $\Sigma$ in a central $\Sigma_{c}(r)$, a vector-isovector $V_{\rho}(r)$ and a Coulomb part $V_{\text {Coul }}(r)$ (only for protons), in the following way

$$
\begin{aligned}
\Sigma(r)_{p, n}= & \frac{\Sigma_{o c}}{1+\exp \left[\left(r-R_{c}\right) / a_{c}\right]} \pm \frac{V_{o \rho}}{1+\exp \left[\left(r-R_{\rho}\right) / a_{\rho}\right]} \\
& +V_{\text {Coul }}(r) .
\end{aligned}
$$

In $V_{\rho}$ the plus sign is for protons and the minus sign for neutrons. The parameters, as functions of $A, N$ and $Z$, are

$$
\begin{aligned}
\Sigma_{o c} & =-69.94 \mathrm{MeV} \\
R_{c} & =1.21 A^{1 / 3} \mathrm{fm} \\
a_{c} & =0.13 A^{1 / 3} \mathrm{fm} \\
V_{o \rho} & =-[0.12(N-Z)+3.87] \mathrm{MeV} \\
R_{\rho} & =[0.03(N-Z)+5.05] \mathrm{fm} \\
a_{\rho} & =[0.007(N-Z)+0.27] \mathrm{fm}
\end{aligned}
$$

The parametrization for $R_{c}$ and $a_{c}$ has a natural justification in view of the known $A^{1 / 3}$ dependence of the nuclear radius. The $V_{o \rho}, R_{\rho}$ and $a_{\rho}$ dependencies on $N-Z$ are also justified since they are proportional to the difference between proton and neutron densities. For $V_{\text {Coul }}$ we take the proton electrostatic potential energy in a uniform spherical charge distribution of charge $Z e$ and radius $R_{c}$

$$
V_{\text {Coul }}(r)=\left\{\begin{array}{lll}
\frac{1}{4 \pi \epsilon_{0}} \frac{Z e^{2}}{2 R_{C}}\left(3-\frac{r^{2}}{R_{C}^{2}}\right) & , & r<R_{C} \\
\frac{1}{4 \pi \epsilon_{0}} \frac{Z e^{2}}{r} & , & r \geq R_{C}
\end{array}\right.
$$

We used in Eq. (12) $R_{C}=1.20 A^{1 / 3}$ which has the same $A$ dependence of the nuclear radius.

We show in Fig. 1 the binding $\Sigma$ potential for neutrons and protons in the $\mathrm{Sn}$ isotopic chain. In the proton case we see explicitly the Coulomb barrier produced by the potential (12).

\section{Isospin dependence of pseudospin symmetry}

Using the nuclear potential presented in Eq. (5) we now display the energy splitting for the two pseudospin doublets $\left[3 s_{1 / 2}-2 d_{3 / 2}\right]$ and $\left[2 d_{5 / 2}-1 g_{7 / 2}\right]$. Firstly we present results for the neutrons and after for the protons where we need to consider also the Coulomb potential.

In Fig. 2 is shown the neutron energy splittings for those pseudospin partners as $A$ changes. In both cases the energy differences increase just slightly as $A$ increases. Since the pair $\left[2 d_{5 / 2}-1 g_{7 / 2}\right]$ is more bound than $\left[3 s_{1 / 2}-2 d_{3 / 2}\right]$, one may notice also that the energy splittings are smaller for levels closer to the the Fermi sea.

In a recent work [8] a systematics was established relating the pseudospin splittings to the Woods-Saxon parameters of the nuclear potentials. The conclusions were that the energy differences become smaller as the magnitude of the well depth $|\Sigma|$ decreases and as the diffusivity (of both $\Sigma$ and $\Delta$ potentials) increases, but grow when their radius increases. From Fig. 1 we see that in the isotope chain the magnitude of the neutron potential well decreases and its diffusivity increases as $A$ increases, thus favoring the pseudospin symmetry. However, the increase of the radius with $A^{1 / 3}$ works in the opposite way and thus essentially cancels the previous effects. Thus, the dependence with $A$ of pseudospin splittings for neutrons is small. 

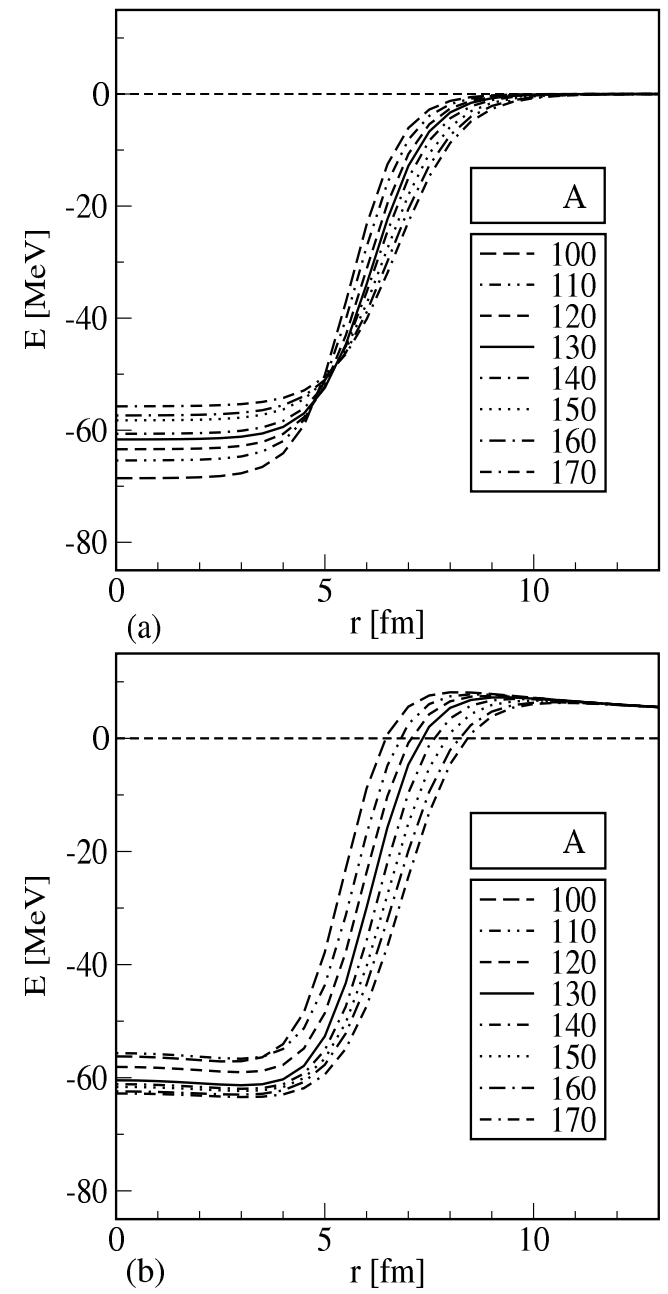

Figure 1. Woods-Saxon potential for neutrons (a) and protons (b) along the $\mathrm{Sn}$ isotopic chain.

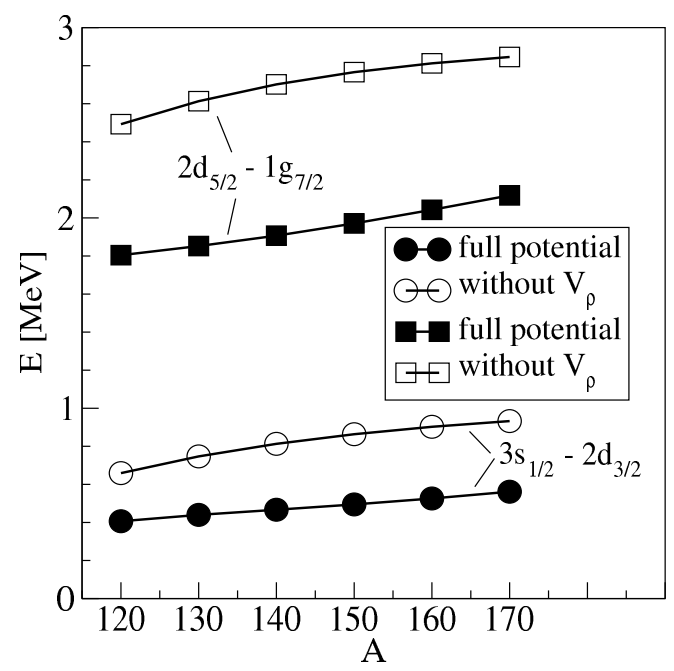

Figure 2. Energy splittings of the pseudospin pairs $\left[3 s_{1 / 2}-2 d_{3 / 2}\right]$ and $\left[2 d_{5 / 2}-1 g_{7 / 2}\right]$ as a function of the mass number $A$ for neutrons with and without $V_{\rho}$.

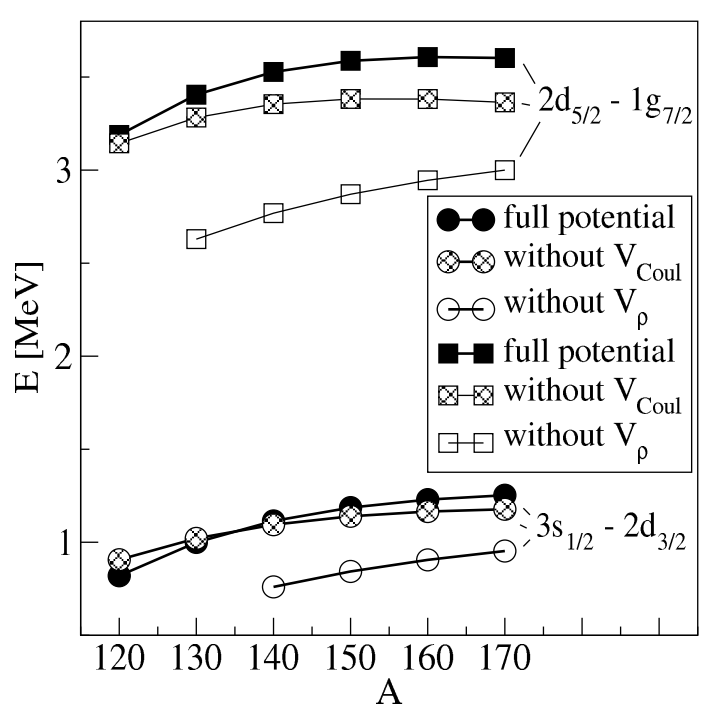

Figure 3. Energy splittings of the pseudospin pairs $\left[3 s_{1 / 2}-2 d_{3 / 2}\right]$ and $\left[2 d_{5 / 2}-1 g_{7 / 2}\right]$ as a function of the mass number $A$ for protons with and without $V_{\rho}$ and $V_{\mathrm{Coul}}$.

We now perform the same study for protons. The result is presented in Fig. 3. Again, we see that there is almost no change of the energy splittings with the mass number $A$. Also, as in the neutron case, the splittings are smaller for higher levels. For example, for $A=150$ the energy difference for the doublet $\left[3 s_{1 / 2}, 2 d_{3 / 2}\right]$ is $1.19 \mathrm{MeV}$ for protons and is $0.49 \mathrm{MeV}$ for neutrons, whereas the $\left[2 d_{5 / 2}, 1 g_{7 / 2}\right]$ pair has an energy difference of $3.58 \mathrm{MeV}$ for protons and of 1.97 $\mathrm{MeV}$ for neutrons. We see that, for the same value of $A$, pseudospin symmetry is favored for neutrons.

What is the origin of this experimentally observed isospin asymmetry of pseudospin in nuclei? Clearly this must be related to both the $V_{\rho}$ potential, which is repulsive for neutrons and attractive for protons, and the Coulomb potential, a repulsive potential which only exists for protons.

Since the Coulomb potential only affects protons, we could think that it could give an important contribution for this asymmetry. However, from Fig. 3 we see that, when the Coulomb potential is turned off, the pseudospin splittings remain practically the same. For example, for $A=150$, if we remove the Coulomb potential, the difference of energy for the doublet $\left[3 s_{1 / 2}, 2 d_{3 / 2}\right]$ is $1.13 \mathrm{MeV}$ (compare with the value of $1.19 \mathrm{MeV}$ given before), whereas for $\left[2 d_{5 / 2}, 1 g_{7 / 2}\right]$ is $3.38 \mathrm{MeV}(3.58 \mathrm{MeV})$.

In [14] we saw that this fact is due to a cancellation between the diverse terms that contribute to the energy of each level. In that study, we showed that, while the contribution of the Coulomb potential to the pseudospin-orbit term is substantial, it is cancelled by the contribution of all the other terms. Fig. 3 shows that, although small, the Coulomb potential contribution is bigger for the pseudospin splitting of deeper pseudospin partners.

In a similar way, we analyze the role of the potential $V_{\rho}$ in the isospin asymmetry by looking into its effect in the splittings of the neutron and proton pseudospin pairs. In Fig. 2 is presented the neutron energy difference of these pairs when the potential $V_{\rho}$ is excluded. When this is done, the energy difference increases, showing that this potential 
favors pseudospin symmetry for neutrons, which can be understood by the systematics referred to before, since $V_{\rho}$, being positive, decreases $|\Sigma|$ (note that $\Sigma<0$ ), and at the same time makes it more diffuse. This can be see in Fig. 1 for the neutrons: $V_{\rho}$ is larger as $A$ and $N-Z$ increases and, as a consequence, the well potential is less profound and more diffuse. For example, again for $A=150$, the energy difference for the neutron doublet $\left[3 s_{1 / 2}, 2 d_{3 / 2}\right]$ without the $\rho$ potential is $0.86 \mathrm{MeV}(0.49 \mathrm{MeV}$ with the full potential), whereas for $\left[2 d_{5 / 2}, 1 g_{7 / 2}\right]$ is $2.76 \mathrm{MeV}(1.97 \mathrm{MeV})$.

In the proton case, shown in Fig. 3 the effect of $V_{\rho}$ is the opposite, as could be expected, but is smaller in magnitude than in the neutron case. This time $|\Sigma|$ becomes bigger when $V_{\rho}$ is added and less diffuse thus working against the realization of the pseudospin symmetry. For $A=150$ the energy difference for the doublet $\left[3 s_{1 / 2}, 2 d_{3 / 2}\right]$ is $0.86 \mathrm{MeV}$ (previously $1.19 \mathrm{MeV}$ ), whereas for $\left[2 d_{5 / 2}, 1 g_{7 / 2}\right]$ is 2.87 $\mathrm{MeV}(3.58 \mathrm{MeV})$. This analysis allows us to conclude that when the vector-isovector potential $V_{\rho}$ is excluded the pseudospin asymmetry for protons and neutrons almost disappears. Therefore, this potential is the main responsible for this asymmetry and the Coulomb potential $V_{\text {Coul }}$ does not play a significant role.

\section{Conclusions}

We have investigated in a quantitative way the isospin dependence of the nuclear pseudospin along the $\mathrm{Sn}$ isotopic chain. To do this analysis we performed a mean-field model calculation with a parametrization for the nuclear potential in this chain developed by us in a recent work [14]. We used Woods-Saxon potentials to fit the proton and neutron potentials obtained by a sophisticated self-consistent calculation for the $\mathrm{Sn}$ isotopes [15].

In this general parametrization of the potential we have separated explicitly the $V_{\text {Coul }}$ and $V_{\rho}$ potentials. These two potentials are the main source of the isospin dependence of the nuclear pseudospin. In order to identify the origin of this dependence we have analyzed in detail the effect of those potentials separately in the proton and neutron pseudospin energy splittings along the $\mathrm{Sn}$ isotopic chain.

We conclude that the dependence with mass number $A$ of the pseudospin symmetry measured by the energy splittings is small either for neutrons or for protons along the isotopic chain. The effect of the Coulomb barrier is also very small and almost negligible for the proton levels close to the Fermi sea. The difference seen in nature for the pseudospin energy splitting of the neutrons and protons comes essentially from the vector-isovector $V_{\rho}$ potential. It makes the binding $\Sigma$ potential more diffuse for neutrons than for protons. Thus the energy splitting decreases for neutrons and increases for protons originating this isospin asymmetry in the nuclear pseudospin.

Finally, from our analysis we can conclude that, at least for tin isotopes, the realization of pseudospin symmetry is almost independent of the neutron content of nuclei in a isotopic chain.

\section{Acknowledgments}

R. L. thanks the nice atmosphere during the XV RETINHA where this work has been presented. P. A. acknowledges the financial support from FCT (POCTI), Portugal. M. M. acknowledges the financial support from the CNPq/ICCTI Brazilian-Portuguese scientific exchange program. M. M and R. L. acknowledge in particular the CNPq support.

\section{References}

[1] K. T. Hecht and A. Adler, Nucl. Phys. A137, 129 (1969)

[2] A. Arima, M. Harvey, and K. Shimizu, Phys. Lett. B 30, 517 (1969)

[3] A. L. Blokhin, C. Bahri, and J. P. Draayer, Phys. Rev. Lett. 74, 4149 (1995)

[4] C. Bahri, J. P. Draayer, and S. A. Moszkowski, Phys. Rev. Lett. 68, 2133 (1992)

[5] O. Castaños, M. Moshinski, and C. Quesne, Phys. Lett. B277, 238 (1992)

[6] J. N. Ginocchio, Phys. Rev. Lett. 78, 436 (1997); ibid, Phys. Rept. 315, 231 (1999)

[7] J. Meng, K. Sugawara-Tanabe, S. Yamaji, P. Ring, and A. Arima, Phys. Rev. C58, R628 (1998)

[8] P. Alberto, M. Fiolhais, M. Malheiro, A. Delfino and M. Chiapparini, Phys. Rev. Lett. 86, 5015 (2001)

[9] P. Alberto, M. Fiolhais, M. Malheiro, A. Delfino, and M. Chiapparini, Phys. Rev. C65, 034307 (2002)

[10] R. Lisboa, M. Malheiro, A. Delfino, P. Alberto, M. Fiolhais and M. Chiapparini, "8th/interaction Workshop on Hadrons Physics 2000; nucl-th 0207068"

[11] S. Marcos, L. N. Savushkin, M. López-Quelle and P. Ring, Phys. Rev. C62, 054309 (2000)

[12] S. Marcos, M. López-Quelle, R. Niembro, L. N. Savushkin, and P. Bernardos, Phys. Lett. B513, 30 (2001)

[13] R. Lisboa, "The dynamical character of the isospin asymmetry in the nuclear pseudospin", dissertation (in portuguese) (Universidade Federal Fluminense, Niterói, 2002) (unpublished)

[14] R. Lisboa, M. Malheiro, and P. Alberto, Phys. Rev. C67, 054305 (2003)

[15] J. Meng and I. Tanihata, Nucl. Phys. A650, 176 (1999)

[16] J. Meng, K. Sugawara-Tanabe, S. Yamaji, and A. Arima, Phys. Rev. C59, 154 (1999)

[17] B. Smith and L. J. Tassie, Ann. Phys. 65, 352 (1971)

[18] J. S. Bell and H. Ruegg, Nucl. Phys. B98, 151 (1975)

[19] J. N. Ginocchio and A. Levitan, Phys.Lett. B245, 1 (1998)

[20] P. Alberto, M. Fiolhais, and M. Oliveira, Eur. J. Phys. 19, 553 (1998) 